\title{
Droplet Populations in Flash Boiling Liquid Nitrogen Sprays
}

\author{
Andreas Rees*1, Lucio Araneo ${ }^{2}$, Heiko Salzmann ${ }^{1}$, Grazia Lamanna ${ }^{3}$, Michael Oschwald ${ }^{1,4}$ \\ ${ }^{1}$ German Aerospace Center (DLR), Institute of Space Propulsion, Langer Grund, \\ Hardthausen 74239, Germany \\ ${ }^{2}$ Politecnico di Milano, Energy Department, Via Lambruschini 4A, Milano 20156, Italy \\ ${ }^{3}$ University of Stuttgart, Institute of Aerospace Thermodynamics, Pfaffenwaldring 31, Stuttgart \\ 70569, Germany \\ ${ }^{4}$ RWTH Aachen University, Institute of Jet Propulsion and Turbomachinery, Templergraben \\ 55, Aachen 52062, Germany \\ *Corresponding author email: Andreas.Rees@dlr.de
}

\begin{abstract}
Technology development for propulsion systems of upper stages and reaction control thrusters is driven by green propellants to substitute hydrazine. At high-altitude conditions prior to ignition the liquid propellants are injected into the combustor at near-vacuum. Due to the sudden pressure drop the liquid is in a superheated thermodynamic state resulting in an eruptive evaporation and fast expansion, a process called flash boiling. To know the composition related to phase and atomization is important for both to determine the probability of a successful ignition and to avoid destructive pressure peaks. Furthermore, the experimental results provide a data base for further numerical investigations about superheated cryogenic fluids. Hence, the cryogenic test bench M3.3 with a temperature-controlled injection system for an experimental investigation of cryogenic flash boiling sprays is in operation at DLR. By means of laser-based Phase Doppler diagnostics the velocity and droplet size distributions of this kind of sprays were determined and two different droplet populations were found. The analysis shows that the flashing sprays are axially symmetrical and that both droplet populations are linked to each other due to similar fluctuations in the transient injection phase.
\end{abstract}

\section{Keywords}

Flash boiling, cryogenic, atomization, Phase Doppler, droplets.

\section{Introduction}

Technology development for propulsion systems of upper stages like the cryogenic Ariane 6 upper stage engine Vinci or for future cryogenic thrusters in reaction control or orbital and manoeuvring systems is driven by the invention of new, green propellants to substitute hydrazine, and by new ignition technologies like laser ignition [1]. At high-altitude conditions prior to ignition the liquid propellants are injected into the combustor at near-vacuum conditions. This means that the ambient pressure $p_{a}$ is lower than the liquid's saturation pressure $p_{s a t}\left(T_{i n j}\right)$ at the injection temperature $T_{i n j}$. The sudden pressure drop at injection leads to a superheated liquid in a metastable thermodynamic state. The injection of a liquid like that results in a fast expansion and eruptive evaporation, a process called flash boiling or flash evaporation. The evaporating gases raise the pressure inside the combustion chamber until the equilibrium pressure shortly before ignition is reached. To know the composition of the propellants in the combustion chamber related to phase, species and temperature distribution is important for determining the parameters for a successful ignition and for avoiding destructive pressure peaks.

\section{Flash Boiling}

The dominating parameters for the flash boiling phenomenon in a given liquid are the injection temperature $T_{i n j}$ and the back pressure $p_{c h}$ which can be a near-vacuum chamber pressure or atmospheric conditions. According to Figure 1, they both define the dimensionless degree of 
superheat $R_{p}$ of the injected liquid in terms of the pressure ratio

$$
R_{p}=\frac{p_{s a t}\left(T_{i n j}\right)}{p_{c h}}
$$

with the saturation pressure $p_{\text {sat }}\left(T_{i n j}\right)$ at the injection temperature $T_{i n j}$ and the back pressure $p_{c h}$. A superheated liquid jet with a high degree of superheat is atomized close to or already in the injector nozzle due to vaporization and produces a fine spray with a big opening angle and small droplets. The influence of aerodynamical processes on the primary atomization can be neglected in these kind of jets [2]. The vaporization and expansion of a flash boiling spray leads to a cool-down to reach a new equilibrium state at the saturation temperature $T_{s a t}\left(p_{c h}\right)$. These non-equilibrium processes are quite complex and need experimental data for further modelling.

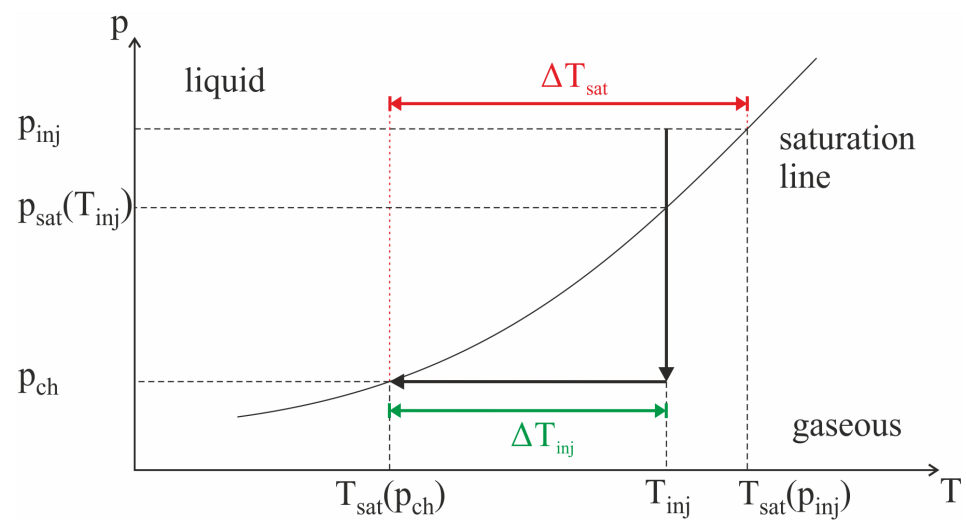

Figure 1. Phase diagram of a superheated liquid for adiabatic depressurization

\section{State of Research}

In the last three decades there have been increased efforts of the automotive industry to investigate flash boiling processes of storable liquids typical for the injection into gasoline or diesel engines $[3,4,5]$. Pre-heating the fuel to reach the superheated condition causes flash boiling during the injection which leads to a finer and wider atomization in the combustion chamber. This increases the efficiency and reduces exhaust emissions [5]. Hydrocarbon sprays with dissolved air were investigated by means of Phase Doppler Anemometry (PDA) and showed similar features like flash boiling sprays [3]. Analytical models were developed for flash boiling hydrocarbon sprays to predict the nucleation rates and the resulting droplet sizes [4] and for water sprays to predict the liquid superheat with the help of the depressurization transient [6]. Further studies about flash boiling processes can be found concerning the safety field in process technology or chemical and nuclear industry, where storable fluids like hydrocarbons, water, ethanol or refrigerants like R-134A were used $[6,7,8]$. It was found that not only the degree of superheat determines the intensity of flash boiling but also injection conditions like the injection pressure or the injector diameter [8]. Transition correlations depending on the dimensionless Weber and Jakob numbers were empirically developed for superheated water leaking into the atmosphere to subdivide the resulting sprays into an aerodynamical break-up region, a transition region and a fully flashing region $[7,9]$. The validity range of these correlations was successfully expanded for the fluids iso-octane, acetone and ethanol [10]. In the same study an onset criterion, which links flash boiling to the classical nucleation theory, for the flashing regimes was developed, and a model for predicting the spray angle in the near-nozzle region by the degree of superheat and the dimensionless surface tension was generated. In contrast to storable fluids, flash boiling of cryogenic liquids is much less investigated due to significantly harder experimental conditions. Within an experimental study at DLR Lampoldshausen about laser ignition in a model rocket combustion chamber at high-altitude conditions, flash boiling was observed for a liquid oxygen (LOX) jet [11]. At the same test bench flash boiling of LOX 
jets with two injection configurations was investigated and the results were compared to flash boiling sprays of storable fluids [12]. Despite the huge differences in their physical properties, the LOX sprays and the sprays with storable liquids showed a similar spray morphology. In another experimental study about cryogenic flash boiling, sprays of liquid nitrogen (LN2) for injection times of about $10 \mathrm{~s}$ were observed with high-speed shadowgraphy for different injection conditions and injector geometries [13]. The resulting sprays showed maximum spray angles of about $140^{\circ}$ and the injector geometry and injection pressure did not have much influence on the spray angles. In our publication [14] a preliminary PDA measurement campaign showed decreasing droplet diameters with increasing injection temperatures in fully flashing LN2 sprays and a global minimum in the vertical velocity profile as a function of the injection temperature. In a consecutive study [15] the droplet velocity and diameter distributions of highly superheated flash boiling LN2 sprays with constant injection conditions were measured by means of PDA and preliminary results like possible recirculation zones or the existence of a second droplet population were shown. A subsequent in-depth data analysis of this measurement campaign showed, that the droplet velocity and diameter distributions of flash boiling LN2 sprays have a sufficient degree of similarity with data of storable fluids from literature [16]. Furthermore, the two different droplet populations were evaluated separately and characteristic features of these distributions could be correlated to flow structures like shock systems due to flash boiling or recirculation zones. In our study [17] the characteristic morphology like break-up patterns and spray angles of flash boiling LN2 sprays was determined by means of high-speed shadowgraphy in dependence on the injection parameters and derived dimensionless quantities. Similar to storable fluids, increasing degrees of superheat lead to a growing dominance of flash boiling on the break-up of the liquid nitrogen jet. Hence, correlations about jet break-up regimes derived for storable fluids showed their suitability for cryogenic nitrogen as well. In addition, a further characteristic flash boiling regime is suggested in that study. In a recent publication [18] it was shown, that existing models to predict the dimensions of the liquid cores in flash boiling LN2 sprays are only applicable for low degrees of superheat.

\section{Experimental Set-Up}

Since the dominating parameters for flash boiling are the injection temperature $T_{i n j}$ and the back pressure $p_{c h}$, it is important for an experimental investigation to make them adjustable, to keep them constant during the injection period and to make them reproducible. Especially adjusting and controlling the temperature was partly limited in the few studies with cryogenic flash boiling. This is why the new test bench M3.3 with a temperature-controlled injection system was built at DLR Lampoldshausen for a detailed experimental investigation of cryogenic flash boiling processes $[14,15,16,17,18]$.

\section{Cryogenic Test Bench M3.3}

The test bench M3.3 consists of three main systems, as depicted in Figure 2: the media supply and pressurization system, the cryogenic temperature adjustment and injection system (CTAIS) and the vacuum system. With the first system all gases (nitrogen, helium) for the operation of the test bench are provided and are pressurized with various pressure reducers to the desired pressures. The second of the three main systems consists of a double-walled and vacuuminsulated pressure tank filled with liquid and gaseous nitrogen (GN2), see Figure 2 on the left and right. By an evacuation or pressurization of the GN2 phase in the pressure tank the fluid is cooled down or heated up, respectively. In the first case a new saturation state is reached due to vaporization of a certain amount of LN2. The latent heat of vaporization necessary for this phase change leads to a loss of heat of the liquid/gaseous nitrogen and consequently to a temperature decrease. In the second case the saturation state after pressurization with GN2 is reached due to condensation of the vapour phase. In this process the latent heat of condensation is released and heats up the nitrogen. Inside the pressure tank is the complete 
LN2 feed and injection system, which consists of a 0.5 liter LN2 run-tank, a coriolis mass flowmeter, the injector unit with a pneumatic run valve and the injector nozzle, and piping inbetween, see Figure 2 in the middle. That means that all these sub-systems are completely surrounded by the cooling medium nitrogen to provide a homogeneous temperature distribution from the run-tank to the injector nozzle. Several dynamic pressure and temperature sensors are installed at the nitrogen pressure tank as well as at the feed and injection system, in order to both control and adjust the temperature of the cooling medium and to measure the injection parameters of the jets. The latter is realized by a Pt100A temperature sensor and a dynamic pressure sensor 601A by Kistler each installed about $30 \mathrm{~mm}$ upstream of the injector nozzle exit. A hand port at the top of the pressure tank provides the feedthroughs for the sensors and the supply pipes for LN2, GN2 and Helium. The latter is used to pressurize the pneumatic axial run valve (Axius by Stöhr Armaturen) as well as the cable ducts for the sensor cables inside the pressure tank. The CTAIS is mounted on top of the vacuum system, which consists of a cylindrical chamber with an inner diameter of $300 \mathrm{~mm}$, a height of $225 \mathrm{~mm}$ from the injector nozzle exit to the bottom of the chamber and four optical accesses with a diameter of $100 \mathrm{~mm}$ each. The four windows are positioned with an angle of $90^{\circ}$ to each other. An attached vacuum pump with a pumping speed of $87.5 \mathrm{~m}^{3} / \mathrm{h}$ produces the near-vacuum atmosphere to simulate high-altitude conditions. After evacuating all of the pipes and vessels of the CTAIS the system is chilled-down in about 90 minutes by filling it with LN2. During the chill-down the run-tank is filled with the gaseous test fluid, which is nitrogen for the current study. The test fluid gets liquefied within this chill-down process. The CTAIS allows variable injection conditions, which can be found in $[14,15,16,17]$. In the first run-in tests we showed that the system is capable of keeping the injection temperature $T_{i n j}$ constant during the whole injection time of about $2 \mathrm{~s}$, that the injection temperature is reproducible in the range of $\pm 0.6 \mathrm{~K}$ for each test run and that the temperature distribution is homogeneous in the whole test fluid feed line [14].

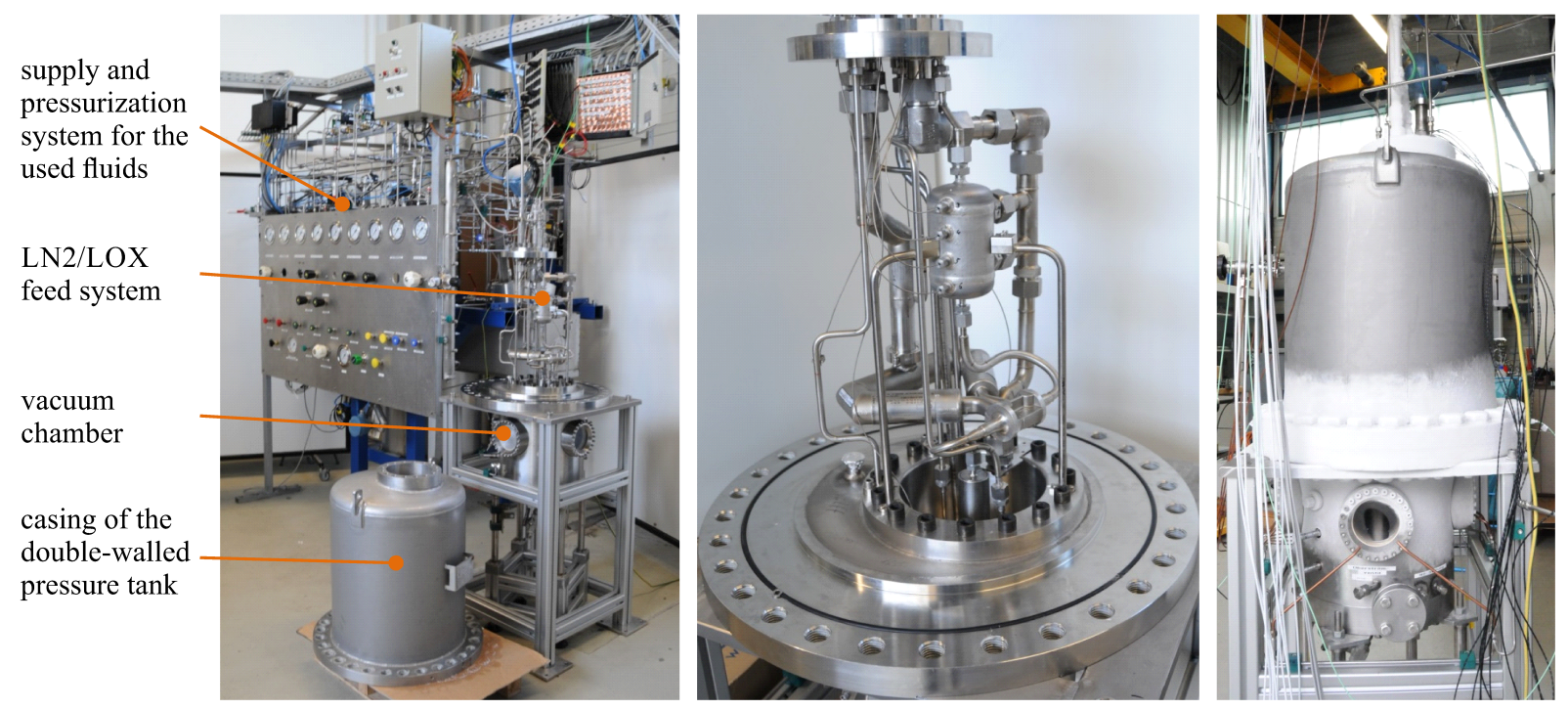

Figure 2. Test bench M3.3 with supply and pressurization system, open CTAIS and vacuum system (left); open CTAIS with run tank, pneumatic run valve, injector unit and sensors in-between (middle); chilled-down test bench in operation mode (right) [16]

\section{Injection Conditions}

We generated highly superheated wide flashing LN2 sprays according to the definition in [16]. Their injection conditions were kept constant and are summarized in Table 1. For all of the generated sprays we used a single sharp-edged injector with a diameter of $D_{i n j}=1 \mathrm{~mm}$ and a length-to-diameter ratio of $L / D=2.9$. Further details about the injection conditions as well as the Weber, Jacob, Reynolds and Ohnesorge numbers of the LN2 sprays can be found in reference [16]. 
Table 1. Injection conditions of the used LN2 sprays

\begin{tabular}{c|cc}
\hline parameter & value & tolerance \\
\hline$T_{i n j}[\mathrm{~K}]$ & 89.7 & \pm 0.6 \\
$p_{i n j}[\mathrm{kPa}]$ & 440 & \pm 40 \\
$p_{\text {ch }}[\mathrm{kPa}]$ & 7 & \pm 2.7 \\
$R_{p}[-]$ & 60 & \pm 39
\end{tabular}

\section{Phase Doppler Diagnostics}

The optical set-up is depicted in Figures 3 and 4 (left) and the main components of the PDA system are summarized in Table 2. Because of the geometrical limited optical access to the spray, we tilted the transmitter and receiver probes and positioned them with an off-axis angle of $\varphi=7^{\circ}$ and $5^{\circ}$, respectively, to use forward scattering first order refraction at a total off-axis angle of $\varphi=12^{\circ}$. Tilting both probes and choosing the total off-axis angle of $\varphi=12^{\circ}$ provides an optimal compromise between the geometrical field of view and the dominant refraction mode. The field of view determines the matrix of measurement positions, which are shown in Figure 4 on the right. We set both DPSS lasers to a power of about $40 \mathrm{~mW}$ which results in a power of approximately $9 \mathrm{~mW}$ for each laser beam in the measurement volume. A spatial filter with a slit of $100 \mu \mathrm{m}$ was chosen. The PDA system was first adjusted and aligned with the help of a water spray generated by a similar nozzle like the one used in the CTAIS. We placed a calibrated needle with a diameter of $1 \mathrm{~mm}$ into the injector nozzle of the CTAIS and illuminated it by one of the laser beams to align the traverse system with the mounted transmitter and receiver probes horizontally. For its vertical alignment we illuminated the lower edge of the injector nozzle with the laser. The refractive index for nitrogen was set to $n=1.205$ according to an interpolation of values derived from reference [19] with respect to the used wavelengths and to the temperature range of the LN2 measurement campaign. With this PDA system we measured the vertical and horizontal droplet velocity as well as the droplet size distributions at different radial and axial locations in fully flashing LN2 sprays at constant injection conditions. Based on the optical set-up of the PDA system, droplets with a maximum particle diameter of $83.2 \mu \mathrm{m}$ can be measured. We set the velocity ranges to $-67 \mathrm{~m} / \mathrm{s} \leq U \leq 203 \mathrm{~m} / \mathrm{s}$ for the vertical velocity component.

Table 2. Components of the optical PDA set-up

\begin{tabular}{c|c}
\hline component & specification \\
\hline 2 DopplerPower DPSS lasers & $1 \mathrm{~W} ; 488 \mathrm{~nm} \& 514 \mathrm{~nm}$ \\
2D transmitter probe & $D=60 \mathrm{~mm} ; \varphi=7^{\circ}$ \\
2D fibre PDA receiver probe & $D=112 \mathrm{~mm} ; \varphi=5^{\circ}$ \\
two front lenses & $f=500 \mathrm{~mm}$ \\
burst processor P800-2D & \\
2D traverse system &
\end{tabular}

For each position of the PDA position matrix on the right-hand side in Figure 4 in terms of the dimensionless radial and vertical coordinates $x / D_{i n j}=x / D$ and $y / D_{i n j}=y / D$, respectively, one single flash boiling LN2 spray was generated and the data were recorded for a duration of $12 \mathrm{~s}$. Data from different positions are thus related to different injection events, where shot-to-shot variations can not be excluded. The start of the data recording is synchronized by the valve opening signal prior to the injection. The systematical measurement error can be quantified to $\pm 26 \%$ for the size determination. Besides this systematical error, the maximum statistical errors can be quantified with $\Delta U= \pm 6.1 \mathrm{~m} / \mathrm{s}$ and $\Delta D_{\text {drop }}= \pm 3.3 \mu \mathrm{m}$ and occur mainly at measurement positions close to the nozzle exit. For further details about the error estimation, we refer to our former publication [16]. 


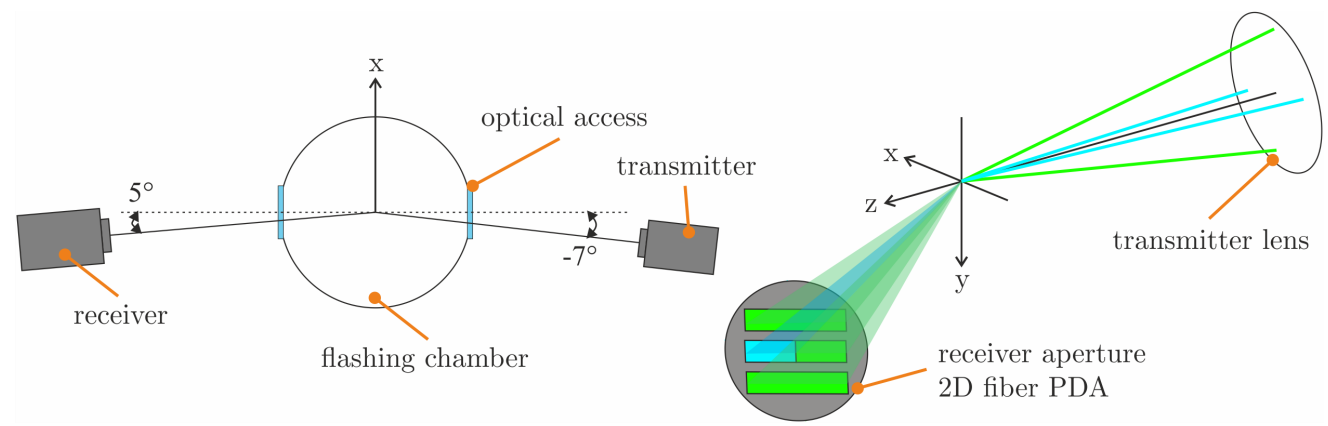

Figure 3. Top view of the optical set-up of the 2D Fibre PDA system centered on the injector symmetry axis at test bench M3.3 (left) and receiver aperture of the used 2D fibre configuration (right) [16]
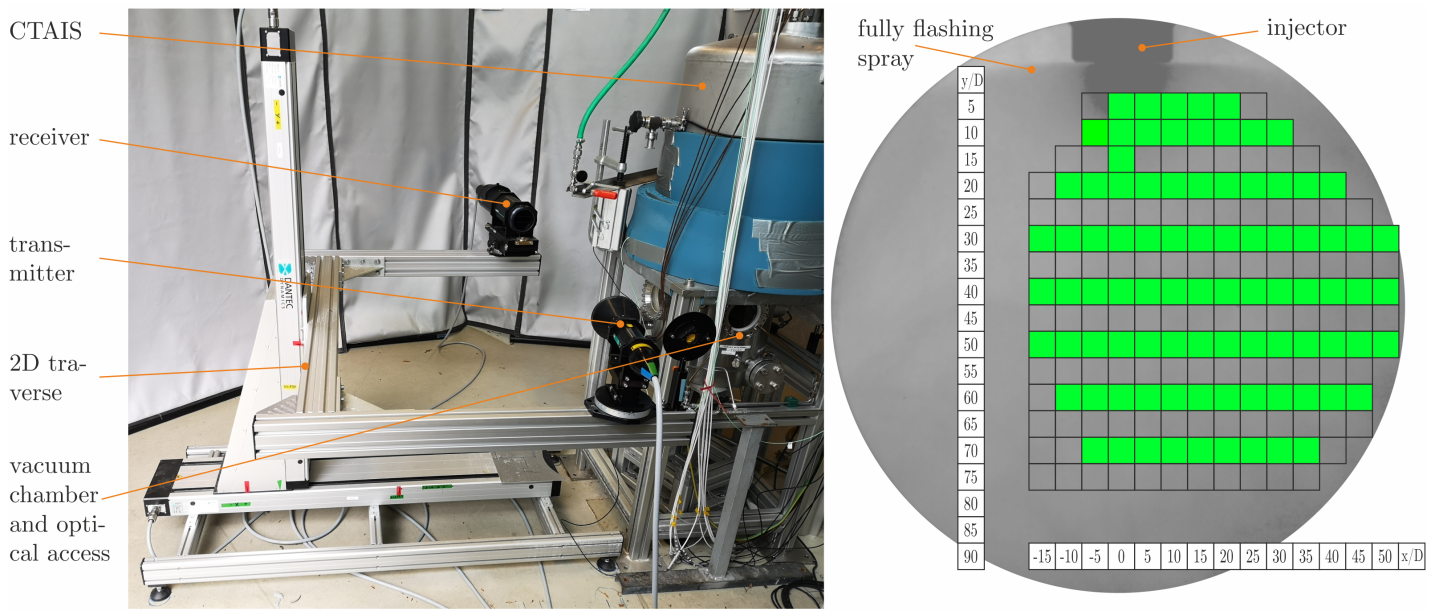

Figure 4. 2D Fibre PDA system at test bench M3.3 (left) and measurement positions in a fully flashing LN2 spray (right) [16]

\section{Results and discussion}

Since the existence and possible reasons of two different droplet populations in flash boiling LN2 sprays have been shown in former works $[15,16]$, a preliminary analysis of the populations themselves is presented in this study. Therefore, the measured vertical droplet velocities $U$ are depicted in the Figures 5 and 6 as functions of the arrival time $t$ at a constant vertical position $y / D=40$ and five different radial positions $x / D=-10,-5,0,5$ and 10.
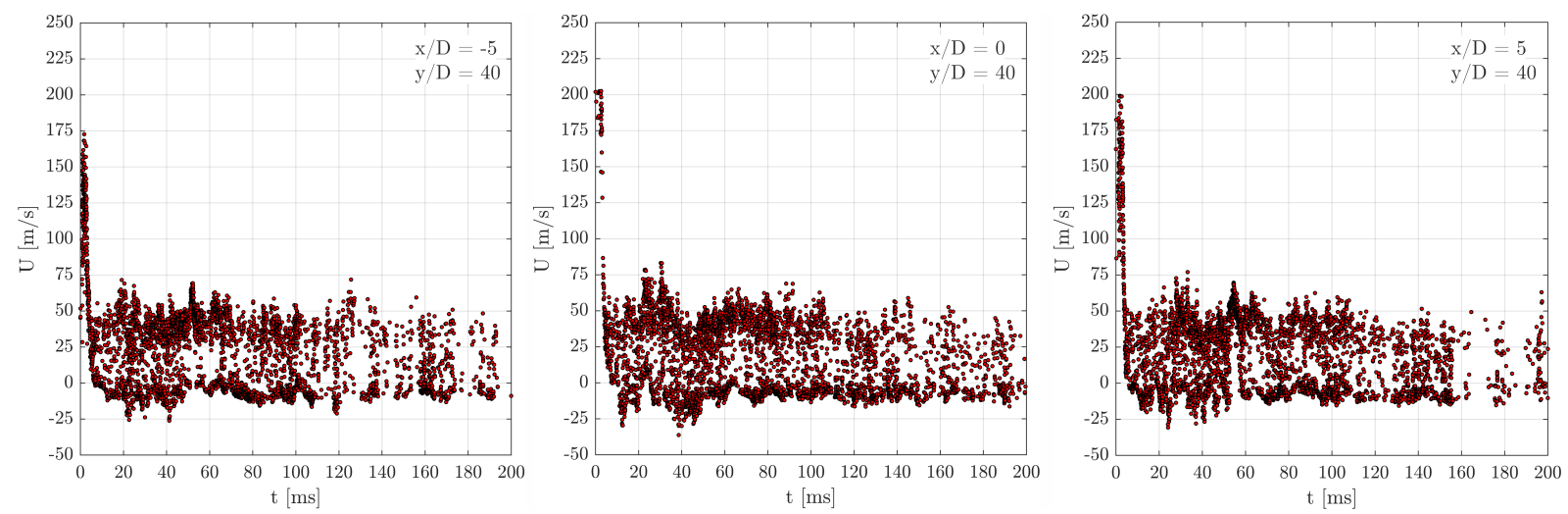

Figure 5. Droplet distribution with the vertical velocity $U$ as a function of the arrival time $t$ in wide flashing LN2 sprays at $T_{i n j}=89.7 \mathrm{~K}$ at the vertical distance $y / D=40$ and the three different radial positions $x / D=-5,0$ and 5

Obviously, all five droplet distributions show the same features with two clearly distinguishable main populations and a high velocity peak at $t<10 \mathrm{~ms}$. Comparing the plots with same distances to the spray's axis of symmetry at $x / D=0$ confirms the previous assumption of 

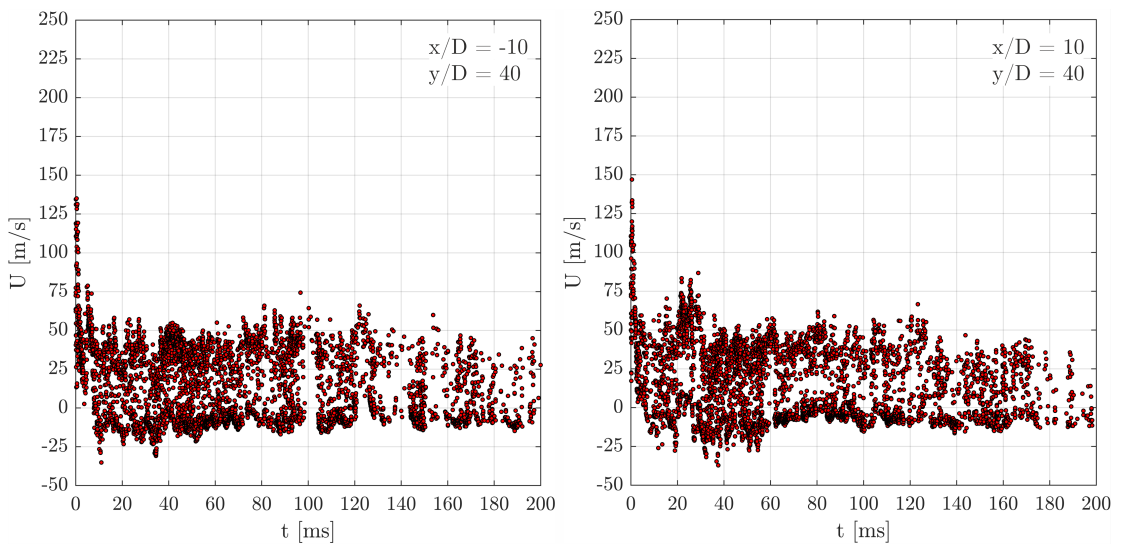

Figure 6. Droplet distribution with the vertical velocity $U$ as a function of the arrival time $t$ in wide flashing LN2 sprays at $T_{i n j}=89.7 \mathrm{~K}$ at the vertical distance $y / D=40$ and the two different radial positions $x / D=-10$ and 10

the axially symmetrical flash boiling process of our LN2 sprays. While close to the centre line the velocities and especially the first velocity peak are reaching values of up to $200 \mathrm{~m} / \mathrm{s}$, they are decreasing towards the sides of the spray. The first peak is caused by the highly dynamic gas flow due to the shock systems typical for flash boiling sprays. These first and fast droplets are very small with measured droplet diameters $D_{\text {drop }}<7 \mu \mathrm{m}$ which is why they can easily follow the initial gas flow. As can be seen, the first peak seems to be connected with the lower droplet population rather then with the upper one with higher droplet velocities of $10 \mathrm{~m} / \mathrm{s}<U<80 \mathrm{~m} / \mathrm{s}$. This confirms the findings in references $[15,16]$ where the low population was linked to the kinematical conditions of the gas flow. Interestingly, the two populations seem to have a kind of crosstalk with each other. This can be seen in terms of similar frequencies of the fluctuations and minor peaks of the two populations during the whole transient injection phase below roughly $100 \mathrm{~ms}$.

\section{Conclusions}

The analysis of the two main droplet populations shows that the flashing sprays are axially symmetrical. Furthermore, both droplet populations appear to be linked to each other due to similar frequencies of the minor droplet velocity peaks and the fluctuations during the transient injection phase. A closer analysis of these frequencies is necessary for their quantification and an explanation of their origin and interaction with each other.

\section{Acknowledgements}

We kindly acknowledge the financial support by the Deutsche Forschungsgemeinschaft (DFG, German Research Foundation) within the project SFB-TRR 75, project number 84292822.

\section{Nomenclature}

$D_{\text {drop }}$ droplet diameter [ $\left.\mu \mathrm{m}\right]$

$D_{i n j} \quad$ injector diameter [mm]

$L / D \quad$ length-to-diameter ratio [-]

$p_{a} \quad$ ambient pressure $[\mathrm{kPa}]$

$p_{c h} \quad$ chamber pressure $[\mathrm{kPa}]$

$p_{i n j} \quad$ injection pressure $[\mathrm{kPa}]$

$p_{\text {sat }} \quad$ saturation pressure $[\mathrm{kPa}]$

$R_{p} \quad$ degree of superheat [-]

$T_{i n j} \quad$ injection temperature $[\mathrm{K}]$

$t \quad$ arrival time [ms]

$t_{i n j} \quad$ time after start signal for injection [s] 
$T_{\text {sat }} \quad$ saturation temperature $[\mathrm{K}]$

$U \quad$ vertical droplet velocity component [m/s]

$x / D \quad$ normalized radial distance $[-]$

$y / D \quad$ normalized vertical distance $[-]$

\section{References}

[1] Manfletti, C., 2014, Laser Ignition of an Experimental 400N Cryogenic Reaction and Control Thruster: Pre-Ignition Conditions, J. Propul. Power, 30 (4), pp. 925-933.

[2] Zeng, Y. and Lee, C.-F.F., 2001, An Atomization Model for Flash Boiling Sprays, Combust. Sci. and Tech., 169 (1), pp. 45-67.

[3] Araneo, L., and Dondè, R., Sep. 2.-4. 2019, Atomization of a G-DI Spray with Air Dissolved in Gasoline and Mono-component Fuels, 29th ILASS-Europe Conference.

[4] Senda, J., Hojyo, Y. and Fujimoto, H., 1994, Modeling on Atomization and Vaporization Process in Flash Boiling Spray, JSAE Review, 15 (4), pp. 291-296.

[5] Senda, J., Wada, Y., Kawano, D. and Fujimoto, H., 2008, Improvement of Combustion and Emissions in Diesel Engines by Means of Enhanced Mixture Formation Based on Flash Boiling of Mixed Fuel, Int. J. Engine Res., 9 (1), pp. 15-27.

[6] Elias, E. and Chambré, P.L., 1993, Flashing Inception in Water During Rapid Decompression. J. Heat Transfer, 115 (1), pp. 231-238.

[7] Cleary, V., Bowen, P. and Witlox, H., 2007, Flashing Liquid Jets and Two-Phase Droplet Dispersion: I. Experiments for Derivation of Droplet Atomisation Correlations, J. Hazard. Mater., 142 (3), pp. 786-796.

[8] Yildiz, D., Rambaud, P, Van Beeck, J. and Buchlin, J.-M., 2002, A Study on the Dynamics of a Flashing Jet, "Final Contract Research Report EAR0030". VKI.

[9] Witlox, H., Harper, M., Bowen, P. and Cleary, V., 2007, Flashing Liquid Jets and TwoPhase Droplet Dispersion: II. Comparison and Validation of Droplet Size and Rainout Formulations, J. Hazard. Mater., 142 (3), pp. 797-809.

[10] Lamanna, G., Kamoun, H., Weigand, B. and Steelant, J., 2014, Towards a Unified Treatment of Fully Flashing Sprays, Int. J. Multiph. Flow, 58, pp. 168-184.

[11] De Rosa, M., Sender, J., Zimmermann, H. and Oschwald, M., Sep. 2.-4. 2006, Cryogenic Spray Ignition at High Altitude Conditions, 42nd AIAA/ASME/SAE/ASEE JPC.

[12] Lamanna, G., Kamoun, H., Weigand, B., Manfletti, C., Rees, A., Sender, J., Oschwald, M. and Steelant, J., 2015, Flashing Behavior of Rocket Engine Propellant, At. Sprays, 25 (10), pp. 837-856.

[13] Luo, M. and Haidn, O.J., 2016, Characterization of Flashing Phenomena with Cryogenic Fluid Under Vacuum Conditions, J. Propul. Power, 3 (5), pp. 1253-1263.

[14] Rees, A., Salzmann, H., Sender, J. and Oschwald, M., July 1.-4. 2019, Investigation of Flashing LN2-Jets in Terms of Spray Morphology, Droplet Size and Velocity Distributions, 8th EUCASS Conference.

[15] Rees, A., Araneo, L., Salzmann, H., Kurudzija, E., Suslov, D., Lamanna, G., Sender, J. and Oschwald, M., Sept. 2.-4. 2019, Investigation of Velocity and Droplet Size Distributions of Flash Boiling LN2-Jets With Phase Doppler Anemometry, 29th ILASS Europe Conference.

[16] Rees, A., Araneo, L., Salzmann, H., Lamanna, G., Sender, J. and Oschwald, M., 2020, Droplet Velocity and Diameter Distributions in Flash Boiling Liquid Nitrogen Jets by Means of Phase Doppler Diagnostics, Exp. Fluids, 61 (182).

[17] Rees, A., Salzmann, H., Sender, J. and Oschwald, M., 2020, About the Morphology of Flash Boiling Liquid Nitrogen Sprays, At. Sprays, 30 (10), pp. 713-740.

[18] Rees, A., Salzmann, H., Sender, J. and Oschwald, M., March 17.-19. 2021, The Evolution of the Liquid Cores in Flash Boiling LN2 Sprays, 7th Space Propulsion 2020+1.

[19] Johns, H. E. and Wilhelm, J. O., 1937, The Refractive Indices of Liquid Oxygen, Nitrogen, and Hydrogen, Can. J. Res., 15 (7), pp. 101-108. 